\title{
Mesoscopic molecular ions in Bose-Einstein condensates
}

\author{
R. Côté ${ }^{1}$, V. Kharchenko ${ }^{2}$, and M. D. Lukin ${ }^{2,3}$ \\ ${ }^{1}$ Physics Department, University of Connecticut, 2152 Hillside Rd., Storrs, Connecticut 06269-3046 \\ 2 ITAMP, Harvard-Smithsonian Center for Astrophysics, 60 Garden Street, Cambridge, MA 02138 \\ 3 Physics Department, Harvard University, Cambridge, MA 02138
}

(November 2, 2018)

\begin{abstract}
We study the possible formation of large (mesoscopic) molecular ions in an ultracold degenerate bosonic gas doped with charged particles (ions). We show that the polarization potentials produced by the ionic impurities are capable of capturing hundreds of atoms into loosely bound states. We describe the spontaneous formation of these hollow molecular ions via phonon emission and suggest an optical technique for coherent stimulated transitions of free atoms into a specific bound state. These results open up new interesting possibilities for manipulating tightly confined ensembles.
\end{abstract}

PACS numbers(s): 33.90.+h, 34.10.+x, 36.40.-c, 51.90.+r

The experimental realization of Bose-Einstein condensation (BEC) in atomic samples [1] have stimulated a new wave of theoretical and experimental studies of degenerate systems in the dilute and weakly interacting regime. A broad range of techniques from atomic physics and optics have allowed the accurate manipulation of ultracold samples of neutral atoms. In particular, various properties of degenerate gases have been explored, such as their excitation modes [2], superfluidity phenomena [3, [4], and controlled vortex creation [5]. Recent studies are probing ultracold atomic systems in which electric charges may play an important role [6]. These include ultracold plasmas [7], ultracold Rydberg gases [8], as well as ionization experiments in BEC [9].

In this Letter, we explore theoretically the behavior of a dilute atomic Bose-Einstein condensate doped with ionic impurities. We show that the polarization interaction in a condensate can lead to the capture of large numbers of atoms into weakly bound states, resulting in the rapid formation of mesoscopically large molecular ions. We study the spontaneous dynamics of the molecular ion formation and show that the degenerate nature of the condensate, as well as the properties of collective excitations (phonons), play an important role. We further describe a coherent optical technique to prepare molecular ions in specific states. Beside the fundamental interest of studying the formation of such large many-body objects, the effects described here may open up new ways to manipulate cold atoms. In particular, the charged and tightly trapped atomic cloud represents a microtrap that could easily be manipulated and "transported" by external fields. Controlled mechanisms to manipulate tightly confined, strongly interacting atoms may also allow for new approaches for quantum information processing and for studies of other fascinating phenomena such as quantum phase transitions. Before proceeding we note an interesting analogy to early studies involving charged impurities in superfluid helium, where electron bubbles and ion "snowballs" were predicted and observed [10].

We consider the situation in which few ions with ul- tralow kinetic energy are introduced into an atomic BEC. This can be realized, e.g., by rapidly ionizing atoms from the condensate using lasers in a process where the ejected electrons carry essentially all the kinetic energy. This would leave the BEC doped with few ions, and the BECion system in a non-equilibrium state. Alternatively, it could be possible to introduce charged impurities via controlled processes involving either a combination of ion and atom traps, or using surface traps on semiconductor surfaces. As a relaxation process, large numbers of atoms from the condensate can be captured into loosely bound states of the polarization potentials, rapidly forming shells of atoms around ions. Such a process occurs spontaneously through collisions of condensate atoms in which atoms are stimulated down into the molecular ion bound state, and the excess energy is carried away by the condensate collective excitations.

We are interested in the limit $T \rightarrow 0$, and for simplicity, we consider a homogeneous BEC with the neutral gas being the parent atom of the doping ion [11. The ion will polarize a nearby atom (separated by a distance $r$ ) and the two will interact via a polarization potential behaving asymptotically as $-C_{4} / 2 r^{4}$, where $C_{4}$ is the dipole polarizability of the neutral atom.

Under the conditions mentioned above, two types of scattering processes involving the ion are possible: elastic and super-elastic collisions. The energy of the colliding partners is not changed in the first type, while kinetic energy is released in the second type. Elastic scattering of an atom and an ion is often described as the contributions from two separate processes [6, 12]: resonant charge transfer scattering (the electron changes center after the collision), and pure elastic scattering (the electron remains with the same center). According to Wigner's threshold laws [13], the rate of all elastic processes vanishes as $T \rightarrow 0$. The second type of scattering process, i.e. super-elastic scattering, corresponds to an inelastic process where one condensate atom is captured by the ion and where kinetic energy is released via the emission of phonons. Here, condensate atoms are accelerated by 
the ionic field (see Fig. 17a), and after the collision, one of the neutral atoms is captured by the polarization potential, and the kinetic energy released is shared by the remaining free condensate atoms and the newly formed molecular ion 14]. Inelastic collisions with excitations of BEC collective modes usually cannot be decribed by simple binary collisions, unless in the limit where the binding energy of the uppermost bound level of the molecular ion is much larger than the chemical potential of the BEC. According to Wigner's threshold laws [13], the rate for super-elastic processes tends to a constant at zero temperature, and this scattering process will be dominant at $T \rightarrow 0$. Contrary to the slow-down of impurities in condensates, where phonon radiation is not possible below some critical velocity (because of momentum-energy conservation (4⿴囗十), the capture of atoms by the long range potential created by the ion corresponds to free-bound transitions, and does not suffer from this restriction: phonon emission takes place at any velocity.

We describe the evolution of the number of atoms $N_{v}$ in the bound level $v$ of the polarization potential by a kinetic equation (see Fig. 1 1 a)

$$
\frac{d N_{v}}{d t}=W_{v}^{\text {cap }}\left(N_{v}+1\right)-\left(W_{v}^{\text {down }}+W_{v}^{\text {up }}\right) N_{v},
$$

where $W_{v}^{\text {cap }}$ is the capture rate from the condensate, and $W_{v}^{\text {down }}$ and $W_{v}^{\text {up }}$ are the loss rates to more deeply bound states and back to the condensate, respectively. The factor $N_{v}+1$ comes from the bosonic nature of the atoms: the capture will be bose-enhanced (or stimulated), as opposed to the depletion of the level (proportional to $N_{v}$ ).

Let us first calculate $W_{v}^{\text {cap }}$. As mentioned above, the capture rate is equal to the phonon emission rate. For Bogoliobov quasi-particles (or phonons), the emission rate of phonons with momemtum $\vec{q}$ and energy $\hbar \omega_{\vec{q}}$ can be estimated using the Fermi Golden rule [4, 15.

$$
w_{\text {emis. }} .(\vec{q})=\frac{2 \pi}{\hbar} \frac{\mu_{c}^{2}}{n V} \frac{\hbar^{2} q^{2}}{2 m} \frac{\left(n_{\vec{q}}+1\right)}{\hbar \omega_{\vec{q}}} \delta\left(\Delta \varepsilon-\hbar \omega_{\vec{q}}\right) I(\vec{q}),
$$

where $V$ is the volume occupied by the condensate, $\mu_{c}=4 \pi \hbar^{2} n a / m$ its chemical potential $(n, m$, and $a$ are the density, mass, and scattering length of the condensate atoms, respectively), and $n_{\vec{q}}$ the phonon occupation number. Here, $I(\vec{q})$ is the square of the form factor for transitions from a continuum to a bound state. If we represent the initial condensate state by the single particle wavefunction $\Psi_{0}(\vec{r})$ and the final bound state by the wavefunction $\Psi_{v}(\vec{r})$, with energy difference $\Delta \varepsilon=\varepsilon_{0}-\varepsilon_{v}=\hbar \omega_{\vec{q}}$, then

$$
I(\vec{q})=N\left|\int d^{3} r \Psi_{v}^{*}(\vec{r}) e^{-i \vec{q} \cdot \vec{r}} \Psi_{0}(\vec{r})\right|^{2},
$$

where $N=n V$ is the number of atoms in the condensate.

At zero-temperature in an infinite homogeneous system, we set $\varepsilon_{0}=0(\vec{k}=0)$, and we have [16]

$$
\Psi_{0}(\vec{r})=\frac{1}{\sqrt{V}} \quad \text { and } \quad \Psi_{v}(\vec{r})=\frac{1}{\sqrt{2 \pi a_{v}}} \frac{e^{-r / a_{v}}}{r},
$$

where the binding energy of the final bound state is related to the extent of the $s$-wavefunction $\Psi_{v}(\vec{r})$ via $\varepsilon_{v}=-\hbar^{2} / 2 \mu a_{v}^{2}$, with $\mu$ being the system reduced mass. Note that we have $\Delta \varepsilon=\hbar^{2} / 2 \mu a_{v}^{2}$. In an isotropic Bose gas, $I(\vec{q})$ depends only on the magnitude of $\vec{q}$, and with

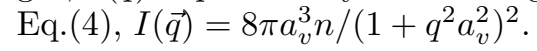

The capture rate is obtained by integrating over all possible phonon states, i.e. $\int d^{3} q w_{\text {emis. }}(\vec{q}) V /(2 \pi)^{3}$, and

$W_{v}^{\text {cap }}=4 \frac{\mu_{c}}{\hbar} \sqrt{\frac{m}{\mu}} \frac{a}{a_{v}} \frac{\left(\sqrt{1+\xi^{2}}-\xi\right)^{3 / 2}}{\sqrt{1+\xi^{2}}}\left(n_{q_{0}}+1\right) I\left(q_{0}\right)$,

where $\hbar \omega_{q_{0}}=\Delta \varepsilon$, and $\xi=\mu_{c} / \Delta \varepsilon=8 \pi n a_{v}^{2} a \mu / m$. For Bogoliobov phonons, $\hbar \omega_{q}=\hbar q s \sqrt{1+(\hbar q / 2 m s)^{2}}$, and we can express $q_{0}$ as

$$
q_{0}^{2} a_{v}^{2} \frac{\mu}{m}=\sqrt{1+\xi^{2}}-\xi
$$

where the sound velocity $s$ is related to the chemical potential by $\mu_{c}=m s^{2}$, and $W_{v}^{\text {cap }}$ becomes

$$
\begin{aligned}
W_{v}^{\text {cap }}= & 4 \frac{\mu_{c}}{\hbar}\left(\frac{m}{\mu}\right)^{3 / 2} \frac{\xi\left(\sqrt{1+\xi^{2}}-\xi\right)^{3 / 2}}{\sqrt{1+\xi^{2}}} \\
& \times\left[1+\frac{m}{\mu}\left(\sqrt{1+\xi^{2}}-\xi\right)\right]^{-2}\left(n_{q_{0}}+1\right) .
\end{aligned}
$$

For infinitly massive ions, we have $m / \mu=1$ [17. Note that the two limiting values of $\xi$ correspond to two different physical conditions: $\xi \rightarrow \infty$ implies $q_{0} \rightarrow 0$, i.e. a phonon-like regime, and $\xi \rightarrow 0$ implies dilute conditions with a binary collision regime. In Fig. 2, we illustrate $W_{v}^{\text {cap }}$ as a function of $\xi$, and show its behavior for the asymptotic regimes: $W_{v}^{\text {cap }} \propto n^{2} a_{v}^{2} a^{2}$ for small $\xi$, and $W_{v}^{\text {cap }} \propto 1 / a_{v}^{3} \sqrt{n a}$ for large $\xi$. The binary regime $(\xi \ll 1)$ is proportional to $n^{2}$ as expected. The $n^{-1 / 2}$ dependence for $\xi \gg 1$ clearly indicates the dominant contribution of phonon assisted transitions [15, 18]. The increase in sound velocity $s \propto \sqrt{n}$ with the condensate density $n$ reduces the rate of phonon emission (and $W_{v}^{\text {cap }}$ [19].

As an example, we consider a sodium ion $\left(\mathrm{Na}^{+}\right)$in a condensate of sodium atoms at $T=0$, with $n \sim 10^{14}$ $\mathrm{cm}^{-3}$ and $a=52 a_{0}$ [20] ( $a_{0}$ : bohr radius). For the uppermost bound state of the polarization potential, $a_{v}$ is well approximated by the atom-ion scattering length $a_{i} \sim 2000 a_{0}$ [6,21], and assuming $m / \mu \approx 1$ [17, we obtain $\xi \sim 0.066$, and neglecting $n_{q_{0}}, W_{v}^{\text {cap }} \sim 600 \mathrm{~s}^{-1}$ (see Fig. 2). Under these conditions, roughly 600 atoms will be captured by the ion, and another 600 will be emitted as phonons (excited out of the condensate) per second. In view of typical condensate lifetimes, this represents a sizable transfer mechanism that should be observable.

By comparison, the capture rate into more deeply bound states will be much smaller. E.g., using the approach of LeRoy and Bernstein [22], we find for the second uppermost level of the pure polarization potential 


$$
a_{v-1}^{2}=\frac{a_{v}^{2}}{1+\left(2 \mu a_{v}^{2} / \hbar^{2}\right)^{1 / 4} K_{4}}
$$

where $K_{4}=4 \sqrt{2 \pi \hbar^{2} / \mu} \Gamma(5 / 4) /\left[\Gamma(3 / 4) C_{4}^{1 / 4}\right]$. Because $a_{v-1}<a_{v} \sim a_{i}$, the capture rate will be accompanied by atom-like excitations with $W_{v}^{\text {cap }} \propto a_{v}^{2}$, and

$$
\frac{W_{v}^{\text {cap }}}{W_{v-1}^{\text {cap }}} \approx \frac{a_{v}^{2}}{a_{v-1}^{2}} \simeq 1+\left(\frac{2 \mu a_{v}^{2}}{\hbar^{2}}\right)^{1 / 4} K_{4} .
$$

For $\mathrm{Na}, C_{4}=162.7$ a.u. [6], and assuming $m / \mu \approx 1$, we find that $W_{v}^{\text {cap }}$ is about ten times larger for the uppermost level than $W_{v-1}^{\text {cap }}$ for the second uppermost level. The ratio is even larger for deeper levels: spontaneous capture is important mostly in the uppermost level.

Let us now examine the escape rates out of the bound states $v, W_{v}^{\text {up }}$ and $W_{v}^{\text {down }}$. Since only the capture in the uppermost level is relevant, we consider only this level. At very low temperatures, $k_{B} T \ll \Delta \varepsilon$, and there are no thermal phonons with sufficient energy to promote bound atoms to the free condensate state, and non-equilibrium phonons emitted from the capture process are moving far away from the location of the ion and can be neglected. Hence, because of energy conservation, transitions from bound levels to the continuum (into the condensate) are not allowed, and $W_{v}^{\text {up }} \sim 0$. The collision of atoms within the upper bound level (or condensate atoms with the trapped ones) may result in the decay of the upper level to a lower level. The rate of this process $\left(W_{v}^{\text {down }}\right)$ is inversely proportional to the binding energy of the deeper level: $W_{v}^{\text {cap }} / W_{v}^{\text {down }} \sim a_{v}^{2} / a_{v-1}^{2}$. Hence, $W_{v}^{\text {down }}$ is of the same order as $W_{v-1}^{\text {cap }}$, and is at least one order of magnitude smaller than $W_{v}^{\text {cap }}$.

As the number of trapped atoms $N_{v}$ within the uppermost bound level $v$ increases, the atom-atom repulsive meanfield energy [23] will grow, and will eventually "push" the energy level up. Using a simple treatment based of the Gross-Pitaevskii equation, one can show that the binding energy of the uppermost level $v$, for $N_{v} \gg 1$, is approximately given by $\varepsilon_{v}\left(N_{v}\right) \simeq\left(m a_{v} / 6 \mu a N_{v}\right)^{2 / 3} \varepsilon_{v}$. Although the number of atoms trapped in the level $v$ would apparently grow to extremely large numbers (since the decay rates out of it are much smaller), other effects, such as charge hopping [6] or thermal fluctuations, will limit that maximum number. The most important one is related to thermal fluctuations. If their energy $\left(\sim k_{B} T\right)$ is equal to $\left|\varepsilon_{v}\left(N_{v}\right)\right|$, thermal equilibrium is reached, and as many atoms get kicked out of the level as there are being captured. In other words, $W_{v}^{\text {up }}$ will be equal to $W_{v}^{\text {cap }}$. At that point, $N_{v}^{\max }$ can be found from $\varepsilon_{v}\left(N_{v}^{\max }\right) \sim k_{B} T$, and $\xi$ will have reached its maximal value $\xi \sim \mu_{c} / k_{B} T$. For a BEC of $\mathrm{Na}$ atoms with $a_{v} \sim R_{c} \sim 2000 a_{0}$ and $T \sim 100 \mathrm{nK}$, we get $N_{v}^{\max } \sim 600$. In Fig. 2, we illustrate the trajectory of the system as a function of $\xi$ for various initial values of $a_{v}$, for both sodium and rubidium condensates. In all cases, as $\xi$ grows from its initial to its final equilibrium value, the capture rate $W_{v}^{\text {cap }}$ passes through a maximum before reaching its final value: then the rates "in" and "out" of the uppermost level are equal, and the system has reached thermal equilibrium.

Lasers can also be used to control coherent stimulated transitions of free condensate atoms into a specific bound state $v$ of the molecular ion. Two off-resonant laser fields with a frequency difference matching the transition energy into a specific bound state $v$ cause a stimulated twophoton transition from the condensate into the bound state of molecular ion (see Fig. 1 $1 \mathrm{~b}$ ). We assume that two laser beams are co-propagating, hence negligible momentum is transfered to the atoms by the lasers. In the limit of sufficiently slow excitation, the atom-laser field interaction is described by $H_{\text {int }}=\hbar \Omega \int d^{3} r \Psi_{v}(\vec{r})^{*} \Psi_{0}(\vec{r}) \hat{b}_{v}^{\dagger} \hat{b}_{0}+$ h.c., where $\Omega$ is the Rabi-frequency of the two-photon transition proportional to the laser intensity, and $\hat{b}_{0}, \hat{b}_{v}$ are bose annihilation operators for the condensate and bound atoms, respectively. The resulting dynamics corresponds approximately to that of a two-component condensate with effective coupling. The number of atoms stimulated into the bound state can be estimated by solving the equations of motion for $\hat{b}_{0}, \hat{b}_{v}$. For a sufficently short interaction time $\tau$, assuming an undepleted condensate $\left\langle\hat{b}_{0}^{\dagger} \hat{b}_{0}\right\rangle=N$, and neglecting spontaneous relaxation, we obtain for $N_{v}=\left\langle\hat{b}_{v}^{\dagger} \hat{b}_{v}\right\rangle$

$$
N_{v}(\tau) \simeq|\Omega|^{2} \tau^{2} I_{v}(0)=8 \pi a_{v}^{3}|\Omega|^{2} \tau^{2} N / V .
$$

The rate of stimulation $W_{v}^{\text {st }}$ is therefore proportional to the laser power with a characteristic frequency scale given by $W_{v}^{\text {st }} \sim\left(\Omega \sqrt{a_{v}^{3} N / V}\right)$, which can be easily made larger than spontaneous capture rate $W_{v}^{\text {cap }}$.

In practice, very fast excitation by lasers might result in heating of the condensate. The heating rate $W_{v}^{\text {heat }}$ is given by the transition probability from the bound state into continuum (Fig. 1 $\mathrm{cc}$ ). This rate is also proportional to the laser power and can be estimated using Fermi's Golden rule. Taking $1 / W_{v}^{\text {st }}$ as a characteristic transition time, we find that heating is negligible in dilute condensate $\left(N a^{3} / V \ll 1\right)$ : $W_{v}^{\text {heat }} \ll W_{v}^{\text {st }}$ provided that

$$
W_{v}^{\text {st }} \ll \hbar / m(N / V)^{2 / 3} \text {. }
$$

For realistic parameters, such as those of a sodium condensate used above, the stimulated rate $W_{v}^{\text {st }}$ can be in the range of $100 \mathrm{KHz}-1 \mathrm{MHz}$ without substantial heating of the condensate. For weakly bound states, such rates are achievable with modest laser powers used, e.g., in Bragg spectroscopy experiments [24]. It is important to note that as the number of bound atoms increases, atomatom interaction can shift the bound state energy out of resonance, thereby inhibiting coherent transitions. This is directly analogous to the recently proposed "dipole blocade" in Rydberg atoms [25], and can be potentially used for non-trivial manipulation of quantum states in a BEC.

In conclusion, we predicted the possiblity of forming large molecular ions in a BEC, analyzed the dynamics of their spontaneous growth and proposed a technique 
for their fast and coherent generation in specific states. Such mesoscopic molecular ions could be observed, e.g., in experiments involving weak ionization of BECs. Of further interest are experiments in which charged impurities are introduced via controlled processes involving either a combination of ion and atom traps, or using surface microtraps. The present work suggests that in such situations, ionic impurities can be used as a new tool for the accurate manipulation of condensates, including their quantum state. Finally we note that one could manipulate, using external electric and magnetic fields, the position of the atomic cloud trapped around a charged particle.

The work of R.C. was supported by the National Science Foundation through the Grant PHY-9970757. The work of V.K. and M.L. was supported by National Science Foundation through ITAMP. This work was also partially supported by MIT-Harvard Center for Ultracold Atoms.

[1] K.B. Davis et al., Phys. Rev. Lett. 75,3969 (1995); M.H. Anderson et al., Science, 269, 198 (1995); C. C. Bradley et al., Phys. Rev. Lett. Phys. Rev. Lett. 75, 1687 (1995); D.G. Fried et al., Phys. Rev. Lett. 81, 3811 (1998).

[2] D.S. Jin et al., Phys. Rev. Lett. 77, 420 (1996); D.M. Stamper-Kurn et al., Phys. Rev. Lett. 81, 500 (1998); E. Timmermans, I. Simbotin, and R. Côté, J. Phys. B 33, 4157- (2000).

[3] A. Leggett, Rev. Mod Phys. 71, S318 (1999); M.R. Matthews et al., Phys. Rev. Lett. 83, 3358 (1999); R Onofrio et al., Phys. Rev. Lett. 85, 2228 (2000).

[4] E. Timmermans and R. Côté, Phys. Rev. Lett. 80, 3419 (1998); A.P. Chikkatur et al., Phys. Rev. Lett. 85, 483 (2000).

[5] M.R. Matthews et al., Phys. Rev. Lett. 83, 2498 (1999); B. P. Anderson et al., Phys. Rev. Lett. 86, 2926 (2001); C. Raman et al., Phys. Rev. Lett. 87, 210402 (2001).

[6] R. Côté and A. Dalgarno, Phys. Rev. A 62, 012709 (2000); R. Côté, Phys. Rev. Lett. 85,5316 (2000).

[7] T.C. Killian et al., Phys. Rev. Lett. 83, 4776 (1999).

[8] W.R. Anderson, J.R. Veale, and T.F. Gallagher, Phys. Rev. Lett. 80, 249 (1998); I. Mourachko et al., ibid. 253.

[9] E. Arimondo, private communication.

[10] A.V. Benderskii et al., J. Chem. Phys. 110, 1542 (1999); M. Rosenblit and J. Jortner, Phys. Rev. Lett. 75, 4079 (1995).

[11] The results obtained here are also valid for atoms and ions of different species (with negligible charge transfer).

[12] E.W. McDaniel, Collision Phenomena in Ionized Gases, 1st ed. (Wiley, New York, 1964).

[13] E.P. Wigner, Phys. Rev. 73, 1002 (1948).

[14] The kinetic energy is shared between phonon and molecular ion: with its rapid growth, the molecular ion becomes very massive and recoil will be negligible.
[15] C. Kittel Quantum Theory of Solids, 2nd revised printing, John Wiley \& Sons (New York, 1987).

[16] Replacing $\Psi_{0}(\vec{r})$ by $\left[\cos \left(R_{c} / r\right)-\left(a_{i} / R_{c}\right) \sin \left(R_{c} / r\right)\right] / \sqrt{V}$, where $a_{i}$ is the atom-ion scattering length and $R_{c}=$ $\sqrt{\mu C_{4}} / \hbar$ is the long range scaling parameter, provides corrections for the presence of the ion. In the phonon regime $(\xi \ll 1)$, this amounts to multiply $W_{v}^{\text {cap }}=$ $4 \mu_{c} / \hbar(2 \xi)^{3 / 2}$ by $\left(\pi R_{c} / 4 a_{i}\right)^{2}+1 / 9 \xi^{2}$ (with $m / \mu=1$ ).

[17] The rapid capture of atoms will ensure the validity of the infinite ion mass approximation $m / \mu \approx 1$.

[18] E.M. Lifshitz and L.P. Pitaevskii, Statistical Physics Part 2, Course of Theoretical Physics, Vol.10, by L.D. Landau and E.M. Lifshitz, Pergamon Press (New York) 1991.

[19] This is analogous to the spontaneous photon emission rate $W \sim 1 / c^{3}$. Here, we have $W_{v}^{\text {cap }} \sim \mu_{c} / s^{3} \sim 1 / \sqrt{n}$.

[20] J. Stenger et al., Phys. Rev. Lett. 82, 2422 (1999); R. Côté and A. Dalgarno, Phys. Rev. A 50, 4827 (1994).

[21] The value of $a_{i}$ is of the order of $R_{c}$ 16].

[22] R.J. Le Roy, Can. J. Phys. 52, 246 (1974).

[23] If the scattering length is negative, the meanfield energy will make the bound level deeper instead.

[24] J Stenger, et al., Phys. Rev. Lett. 82, 4569 (1999).

[25] M.D. Lukin et al., Phys. Rev. Lett. 87, 037901-1 (2001).

a)

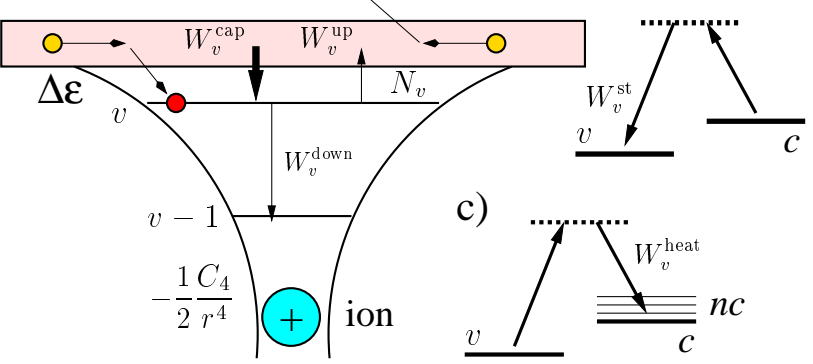

FIG. 1. Diagrams of atom capture by an ion. In (a), the spontaneous capture in level $v$ is followed phonon emission (with corresponding rates). In (b), the stimulated rate from the condensate $c$ to the bound level $v$, also may produce heating, i.e. population of noncondensate atoms $n c$, in (c).

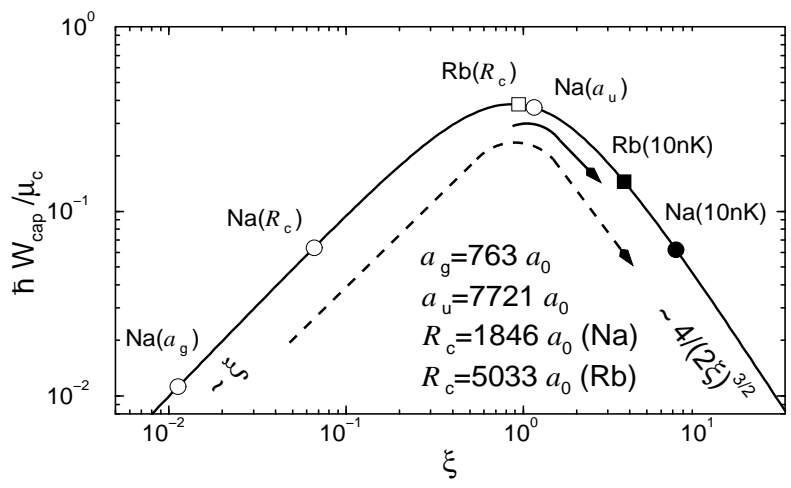

FIG. 2. $W_{v}^{\text {cap }}$ as a function of $\xi$ for various values of $a_{v}$ corresponding to the states ${ }^{2} \Sigma_{g}^{+}$, and ${ }^{2} \Sigma_{u}^{+}$of $\mathrm{Na}_{2}^{+}$[6], as well as $a_{v} \sim R_{c}$ for both $\mathrm{Na}$ and $\mathrm{Rb}$. The equilibrium points for $\mathrm{Na}$ and $\mathrm{Rb}$ at $10 \mathrm{nK}$ are shown, as well as the two limiting behaviours. 